\title{
Numerical investigation of a Mach 9 oblique detonation engine with fuel pre-injection
}

\author{
Zijian Zhang $^{\mathrm{a}, \mathrm{b}}$, Kaifu Ma ${ }^{\mathrm{a}, \mathrm{b}}$, Wenshuo Zhang ${ }^{\mathrm{a}, \mathrm{b}}$, Xin Han ${ }^{\mathrm{a}, \mathrm{b}}$, Yunfeng Liu ${ }^{\mathrm{a}, \mathrm{b}, *}$, \\ Zonglin Jiang ${ }^{\mathrm{a}, \mathrm{b}}$
}

a School of Engineering Science, University of Chinese Academy of Sciences, Beijing 100049, China

${ }^{\mathrm{b}}$ Institute of Mechanics, Chinese Academy of Sciences, Beijing 100190, China

\section{A R T I C L E I N F O}

\section{Article history:}

Received 3 October 2019

Received in revised form 29 January 2020

Accepted 1 March 2020

Available online 2 July 2020

Communicated by Zhao Dan

\section{Keywords:}

Oblique detonation engine

Pre-injection

Detonative combustion

Mixing

Hydrogen

\begin{abstract}
A B S T R A C T
In this study, the performances of a Mach 9 oblique detonation engine fueled by hydrogen are numerically investigated by solving the multi-species reactive Reynolds-averaged Navier-Stokes (RANS) equations with a detailed combustion mechanism. The fuel is perpendicularly pre-injected into the core airflow in the engine inlet by three parallel strut-injectors. It is demonstrated that mixing can be enhanced by the baroclinic effect of oblique shock waves, the incipient expansion of fuel jets and the intensive momentum exchange of vertical jets into the crossflow, resulting in a well-mixed fuel-air core flow before entering the combustor. Analyses of the two most dangerous zones that bear potential of preignition suggest that no pre-combustion occurs in the inlet. Benefiting from the floor bleed structure, the upstream movement of the shock waves stops at the combustor's entrance and they remain stabilized in the combustor thereafter. Finally, the combustor is proved to work under the stable detonation mode of combustion, and the supersonic fuel-air mixture is fast burnt through the steady detonation waves generated in the combustor. The concept of the pre-injection oblique detonation engine has been numerically demonstrated, which provides a significant reference to further experimental studies and future engineering applications.
\end{abstract}

(C) 2020 Elsevier Masson SAS. All rights reserved.

\section{Introduction}

Oblique detonation wave (ODW) is a shock wave that can be stabilized in a supersonic fuel-air combustible mixture by a wedge or a cone [1-3]. Fast combustion is induced by the ODW and completed through a thin detonation front. In recent years, the idea of using oblique detonative combustion in high efficiency propulsion systems, which prompts the concept of oblique detonation engines (ODEs), has attracted great interests in the development of hypersonic air-breathing aircrafts [4-6]. Not only the advantages of scramjets but also the high thermal cycle efficiency through the detonation mode of combustion can be achieved by the ODEs. Moreover, benefiting from the fast shock-induced combustion, the massive combustion chamber of the scramjet can be avoided in the ODE, and hence a large amount of engine weight can be reduced. However, regardless of the great superiorities of this type of engine in hypersonic air-breathing propulsion systems, many technical details of the ODE are greatly desired to be resolved be-

\footnotetext{
* Corresponding author at: Institute of Mechanics, Chinese Academy of Sciences, Beijing 100190, China.

E-mail address: liuyunfeng@imech.ac.cn (Y. Liu).
}

fore it becomes a reliably workable engine, especially the following two main technical challenges: the first is to produce a well premixed combustible supersonic flow before entering the combustor and prevent pre-combustion, especially in the hot boundary layer; the second is to initiate and stabilize a detonation wave in the combustor [7-10].

In the past decades, many efforts have been made to understand the initiation and stabilization of ODWs, through experiments or numerical simulations. Kasahara et al. [11,12], Higgins et al. [13] and Verreault et al. [14] launched hypervelocity projectiles, either spherical or conical in shape, into premixed combustible gases, and steady ODWs around the projectiles were obtained. The detailed ODW structure consisting of an oblique shock wave (OSW), a transverse shock wave and an ODW was experimentally found by Morris et al. [15], using an expansion tube facility, and by Viguier et al. [16], using a two-layer oblique shock tube facility. Two typical initiation structures of wedge-induced ODWs, namely the smooth and abrupt OSW-ODW transitions, were numerically investigated by Fang et al. [17], Tian et al. [18] and Zhang et al. [19], and the effects of wedge angle, incident Mach number, inflow pressure, dilution ratio, etc. on the types of OSW-ODW transitions were clarified, which are helpful to the ODE designs. The instability 


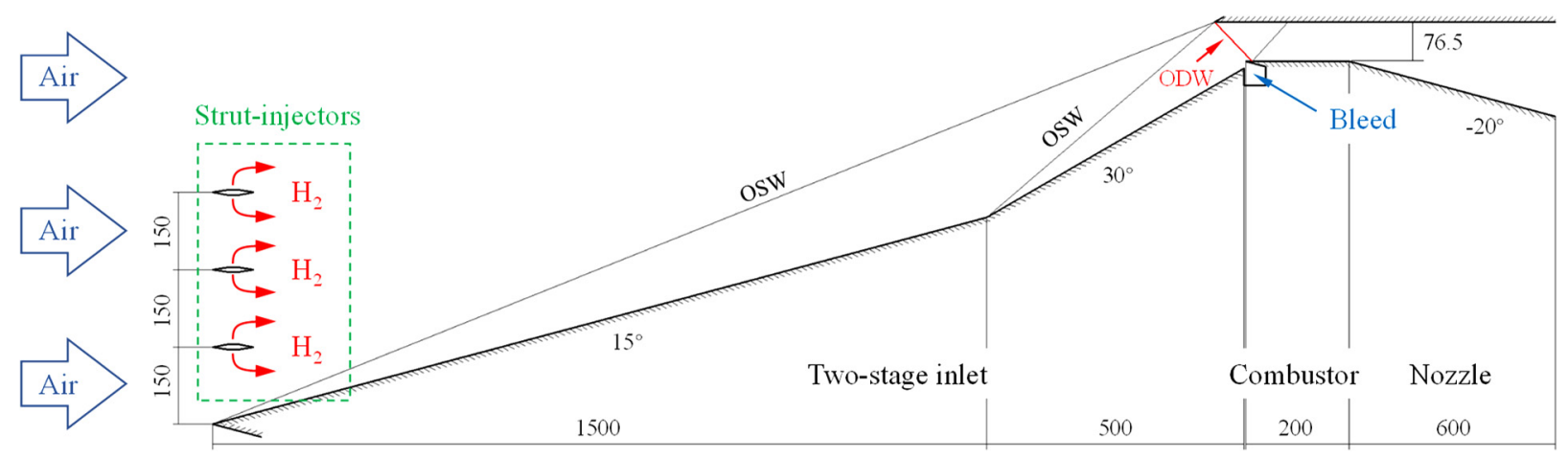

Fig. 1. Schematic of the designed ODE (dimension in $\mathrm{mm}$ ).

on the oblique detonation surfaces and the corresponding oblique cellular detonation structures have been numerically investigated by Liu et al. [20], Teng et al. [21] and Zhang et al. [22]. Both leftrunning and right-running transverse waves have been found, and the effects of inflow Mach number and activation energy on the existence of these two groups of transverse waves have been identified. Numerical simulation was also performed to investigate the effect of boundary layer on oblique detonation initiation by Fang et al. [23], and significant discrepancies, comparing to the inviscid counterparts, of the abrupt OSW-ODW transition structure have been found. For practical applications, wedge-induced ODWs using liquid hydrocarbon fuels and the effects of multiphase characteristics on the stabilization of ODWs have been numerically discussed by Ren et al. [24,25]. The flow field and its evolution of the inviscid ODW within a confined combustor have also been studied by Lu et al. [26].

With the developing understanding of ODWs, it is time to solve the specific challenges of the ODEs and promote their engineering applications. Two configurations of ODEs distinguished by different ways of producing supersonic fuel-air mixture, can be found in literatures [27-32]. The first one, also known as the pre-injection ODE, is the original configuration of ODEs that can trace back to 1940s [27]. The fuel is pre-injected in the inlet of engine and mixed with the incoming airflow while being compressed. Then, the combustion of this fuel-air mixture is induced by an OSW generated in the combustor, and consequently, an ODW is formed. The high-temperature combustion products finally expand in the nozzle to generate the thrust. The second configuration is the so-called shock-induced combustion ramjet (shcramjet), which operates very similar with scramjets [28]. The fuel is injected in the isolator, and the combustion of fuel-air mixture is induced by an OSW located at the end of combustor. Followings are the expansion of the combustion products and the thrust generation as well. Due to the extremely strict requirements of experimental conditions and the great difficulties in experiments, rarely experimental results of ODEs have been reported, which makes the numerical simulation be a rather important, useful and effective means to study the flow mechanism of ODEs and their further designs. A number of numerical work on the second configuration of ODE, that is the shcramjet, has been done by Alexander and Sislian et al. (e.g. Refs. [28-30]). The ideas of using an array of cantilevered ramp injectors to provide fuel in the isolators and of enhancing mixing through the generated streamwise vortexes from injectors, have been proved capable of producing a well-mixed supersonic fuel-air mixture and avoiding pre-combustion in the hot boundary layer. Well combustion of the fuel-air mixture has been achieved by OSW-inducing as well. However, only the shock-induced combustion is implemented in this type of ODE, and the real detonation mode of combustion seems not to be achieved. The reason may be that the induction length for ODW initiation is rather large but the combustor is narrow. The expansion effects caused by the noz- zle immediately after the wedge may also be served as one of the factors $[33,34]$.

As for the pre-injection configuration of ODE, Sislian et al. [31] and Wang et al. [32] also adopted the similar cantilevered ramp injectors to pre-inject fuel into the ODE inlet in their simulations, and well-mixed supersonic fuel-air mixtures and pre-combustion prevention have been achieved as well. However, no further results have been reported by them about stably detonating such fuel-air mixtures in a confined ODE combustor rather than in a semiinfinite space. Boundary layer separations can be induced through detonation wave-boundary layer interactions, and the strength of ODW is always large enough to result in severe separations. Motions or oscillations of ODWs in the combustor and even unstart of the engine may occur, which can lead to the failure of the engine. This is an important issue that needs to be considered carefully when ODWs are applied in internal flows. Therefore, it is imperative to demonstrate the concept of the pre-injection ODE via numerical simulations with all the key technical challenges taking into account (including mixing, pre-ignition prevention, initiation of ODWs and stability of combustion), and to provide a workable solution of the pre-injection ODE for further experimental studies and future engineering applications.

The remaining parts of this paper are organized as follows. In Section 2, the geometry of a simple pre-injection ODE, fueled by hydrogen $\left(\mathrm{H}_{2}\right)$ and designed at Mach 9 , is proposed. Next, in Section 3, some numerical details and the validations of the numerical methods are briefly introduced. Then, the flow field in the ODE inlet and the mixing effect of the proposed fuel pre-injection configuration are analyzed in Section 4.1. The possibility of precombustion in the inlet is investigated in Section 4.2. The flow field in the ODE combustor and the corresponding combustion mode are discussed in Section 4.3. Finally, a conclusive summary is given in Section 5.

\section{Geometry of ODE}

The schematic of a pre-injection ODE, designed at Mach 9, is shown in Fig. 1. The engine is designed to be two-dimensional (2D) in geometry as far as possible, except for the strut-injectors. The engine, composed of three strut-injectors, a two-stage inlet, a combustor and a nozzle, is about $2.8 \mathrm{~m}$ in length and $0.8 \mathrm{~m}$ in height. Notably, this study focuses on the demonstration of producing a well-mixed fuel-air mixture without pre-combustion and the implementation of stable detonative combustion in an ODE. A simple two-stage inlet, composed of a $15^{\circ}$-inclined ramp with $1.5 \mathrm{~m}$ in length and a $30^{\circ}$-inclined ramp with $0.5 \mathrm{~m}$ in length, is employed to compress the inflow without further optimization considerations. The combustor is $0.2 \mathrm{~m}$ in length and $0.0765 \mathrm{~m}$ in height. Obviously, the ODE combustor is rather short even operating at a high Mach number comparing to that of scramjet, which is one of the advantages of ODEs. Following the combustor is an 

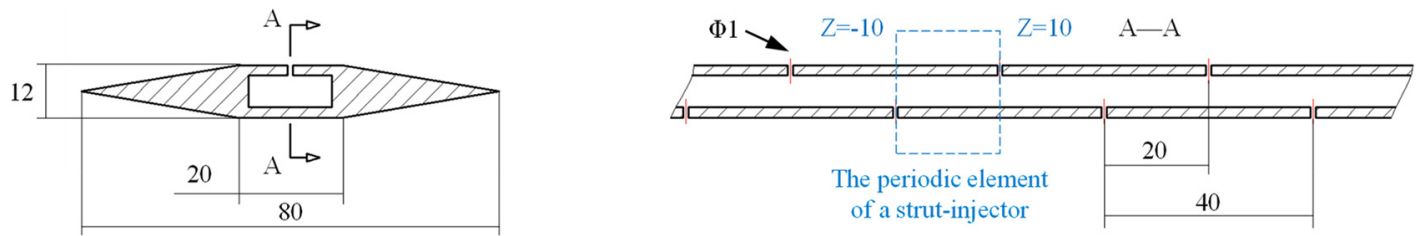

Fig. 2. Schematic of a strut-injector (dimensions in $\mathrm{mm}$ ).
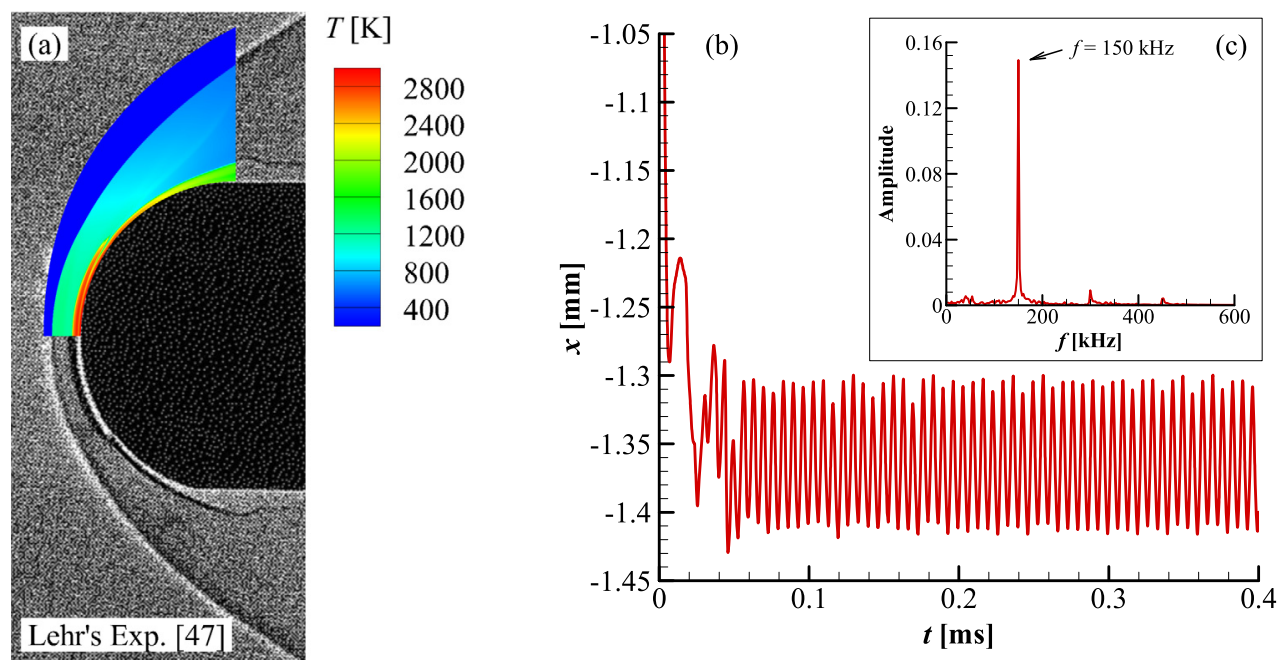

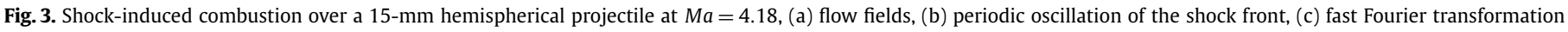
(FFT) result. (For interpretation of the colors in the figure(s), the reader is referred to the web version of this article.)

incomplete nozzle with a $20^{\circ}$ expansion at one side and a length of $0.6 \mathrm{~m}$. Similarly, the optimization of generating thrust by the nozzle is not considered. Further, it has been proven in Ref. [35] that the incomplete nozzle would not influence the performances of the combustor.

It is easy to know that the well-mixing and the pre-combustion prevention in the inlet are contradictory with each other. The traditional configuration of pre-injecting fuel from the inlet wall always results in the existence of a large amount of fuel in the hot boundary layer that bears danger of pre-ignition. Therefore, to reduce the amount of fuel in the hot boundary layer and to take the advantage of the inlet's length, the fuel is designed to be preinjected in the leading front of the inlet, resulting in injecting fuel beyond the first OSW. For simplicity, as shown in Fig. 1, three strut-injectors that are parallel to the inflow and $15 \mathrm{~cm}$ spacing in the vertical direction with each other, are employed in the present design. The detailed structures of the strut-injector are shown in Fig. 2. Arrays of fuel holes, which are $1 \mathrm{~mm}$ in diameter and $2 \mathrm{~cm}$ spacing from each other, are designed staggered on both sides of the strut to provide vertical $\mathrm{H}_{2}$ jets in the inlet. Sonic throats are assumed at the injection holes, and the flow rate of fuel and consequently the equivalence ratio of fuel are uniquely determined by the supplied pressure of fuel. Moreover, the large boundary layer separation induced by the detonation wave-boundary layer interaction is one of the most important factors of the thermal chocking and unstart of the engine combustor. Hence, to control the boundary layer separations in the combustor, a simple floor bleed structure, similar to that in HyShot II [35], is also designed at the entrance of the combustor.

\section{Numerical details}

Both 2D and 3D multi-species reactive Reynolds-averaged Navier-Stokes (RANS) equations are numerically solved by the finite-volume method. The numerical flux through each cell face is evaluated using a second-order total variation diminishing (TVD) scheme based on an approximate Riemann solver named HartenLax-van Leer contact (HLLC) [36,37]. A minmod limiter is used to suppress spurious oscillations near the discontinuities while the high-order accuracy is retained away from the jumps. The integration in time-direction is implicitly implemented via a dual time-stepping method. Moreover, the SST $k$ - $\omega$ turbulence model [38] with a compressibility correction is used to close the RANS equations, and an advanced two-layer wall function $[39,40]$ with an application range of $0.1<y^{+}<300$ is used to model the nearwall flow. The Jachimowski mechanism [41], which involves 19 reversible elementary reactions among 9 species $\left(\mathrm{H}_{2}, \mathrm{H}, \mathrm{O}_{2}, \mathrm{O}, \mathrm{OH}\right.$, $\mathrm{HO}_{2}, \mathrm{H}_{2} \mathrm{O}_{2}, \mathrm{H}_{2} \mathrm{O}$ and $\mathrm{N}_{2}$ ) is employed to model $\mathrm{H}_{2}$ combustion. All species are treated as real gases and their corresponding thermodynamic properties are evaluated from the 9-coefficient NASA polynomial representation [42]. The Jachimowski mechanism predicts ignition delay times in good agreements with experiments [43] and has been successfully applied in the previous scramjet, shock-induced combustion and oblique detonation simulations [35,44-46].

To validate the numerical methods, the classical unsteady shock-induced combustion experiment [47] over a hypervelocity projectile with a Mach number of $M a=4.18$ is numerically tested in this study. The projectile, which had a hemispherical head body with a diameter of $15 \mathrm{~mm}$, was shot into the stoichiometric $\mathrm{H}_{2}$ air mixture with a pressure of $p=0.421 \mathrm{~atm}$ and a temperature of $T=292 \mathrm{~K}$. The comparison of the flow fields between the present simulation and the experiment is shown in Fig. 3a. The flow field obtained by the present simulation agrees well with the experiment. Moreover, as shown in Fig. 3b, the shock-induced combustion in this case is unsteady and oscillates periodically. The oscillation frequency obtained in experiment is about $148 \mathrm{kHz}$, while it is $150 \mathrm{kHz}$ in the present simulation (Fig. 3c). Good agreement is also observed. Another test case is the supersonic combustion experiment conducted by Burrows et al. [48], where 
(a)

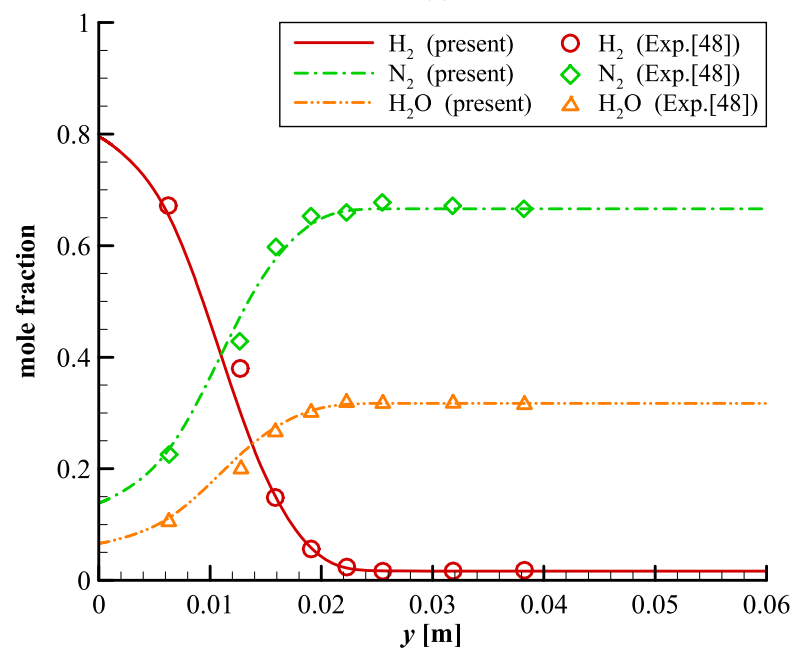

(b)

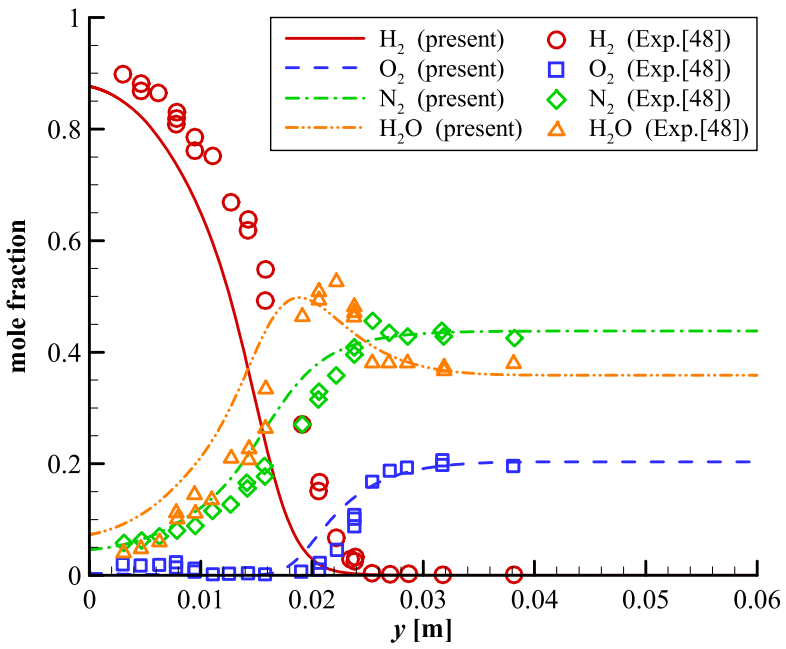

Fig. 4. Mole fraction profiles at the exit of the Burrows' supersonic combustor, (a) mixing case and (b) reacting case.

Table 1

Flow parameters of the freestream and fuel jets used in present simulations.

\begin{tabular}{lll}
\hline & Air & $\mathrm{H}_{2}$ \\
\hline$p_{0}$ & $3.89 \mathrm{MPa}$ & $787 \mathrm{kPa}$ \\
$T_{0}$ & $3572 \mathrm{~K}$ & $300 \mathrm{~K}$ \\
$M a$ & $8.67^{\mathrm{a}}$ & 1.01 \\
Turbulence intensity & $2 \%$ & - \\
\hline
\end{tabular}

a The decrease of freestream Mach number is attributed to the boundary layer in the tunnel nozzle.

a sonic hydrogen jet was parallelly injected into a main stream of $M a=2.44$. The compositions (in mole fraction ratio) of the main streams for the mixing and reacting cases are $\mathrm{H}_{2}: \mathrm{O}_{2}: \mathrm{N}_{2}: \mathrm{H}_{2} \mathrm{O}=$ $0.017: 0: 0.666: 0.317$ and $0: 0.203: 0.438: 0.359$, respectively. Fig. 4 shows the comparisons of the mole fraction profiles at the exit of the combustor. It is shown that the present simulation results agree with the experimental data of Burrows et al. [48] for both the mixing and reacting cases. Therefore, it can be concluded that the above numerical methods are capable of capturing the features of supersonic turbulent mixing, supersonic turbulent combustion and shock-induced combustion.

The ODEs are expected to operate at a Mach number from 8 to 15 and at an altitude from $30 \mathrm{~km}$ to $60 \mathrm{~km}$. In the present simulations, the freestream parameters, as shown in Table 1, are taken as the parameters of a Mach 9 airflow provided by the JF-12 wind tunnel $[49,50]$, which duplicates the flight conditions corresponding to an atmosphere altitude of $45 \mathrm{~km}$. The nominal equivalence ratio of fuel is set as 1.2. Therefore, the supplied sonic $\mathrm{H}_{2}$ jets are assumed to be of a total temperature of $300 \mathrm{~K}$ and a total pressure of $787 \mathrm{kPa}$. All wall boundaries are assumed to be no-slip, noncatalytic and isothermal at $300 \mathrm{~K}$. The supersonic outlet condition is employed at the outlet of the nozzle, while the characteristicsbased low back pressure condition (10 Pa) is imposed to the outlet of the floor bleed structure.

Depending on the unique geometry of the present ODE design, the flow field is 3D near the strut-injectors in the leading front of the engine inlet and it is nearly $2 \mathrm{D}$ from the tail of the inlet to the nozzle-end of the engine. Moreover, the flow in the inlet is thought to be characterized by mixing but without combustion, while the flow in the combustor is characterized by ignition and combustion. Therefore, to obtain the flow field and evaluate the performances of the ODE in an effective way, the total simulation process can be simply divided into three steps. Firstly, assuming no combustion occurs in the inlet, the 3D flow field in the inlet with staggered $\mathrm{H}_{2}$ jets is calculated without chemical reactions to simulate the compression and mixing processes. Secondly, to confirm the no-combustion assumption of the inlet in the first step, the most dangerous zones that possess possibility of pre-ignition are simulated with the detailed chemical reaction mechanism. Finally, the parameters of the outflow of the inlet obtained in the first step are served as the inflow conditions for the 2D reactive simulation of the combustor and the nozzle.

In the 3D simulation of the inlet flow, taking the advantages of the periodicity of the staggered $\mathrm{H}_{2}$ jets and the 2D geometry of the designed ODE model, the computational zone of the ODE inlet is set as $2 \mathrm{~cm}$ in width, which consists of halves of the jets on the both sides of the injectors. The periodic element of one injector is shown by the dashed box in Fig. 2. Structured hexahedral grids are used in this simulation, and the grids are densified near the walls, injection holes and wedge turning corners. The minimum grid size is set as $0.05 \mathrm{~mm}\left(y^{+} \sim 1.5\right)$, which results in about 32 million grids totally.

In the 2D simulation of the combustor flow, quadrilateral meshes are used, and the meshes are also densified near the walls, cowl tip and wall turning corners. The minimum mesh size is set as $0.1 \mathrm{~mm}\left(y^{+} \sim 21\right)$, resulting in about 1.68 million meshes totally. At the beginning, the 2D combustor flow field is initialized with low vacuum stationary air ( $p=10 \mathrm{~Pa}$ and $T=300 \mathrm{~K}$ ), and then the simulation is started with the imposition of the outflow parameters of the engine inlet as the combustor inflow conditions.

\section{Results and discussions}

\subsection{Mixing in $O D E$ inlet}

Fig. 5 shows the distributions of $\mathrm{H}_{2}$ equivalence ratio $(\phi)$ and temperature along with iso-lines of pressure in the convergent $3 \mathrm{D}$ flow field of the ODE inlet at the slice of $z=0$. With $\mathrm{H}_{2}$ preinjection into the airflow, the flow field is quite different from that without fuel injection, and complex shock waves can be observed. Aside from the main compression OSWs generated by the twostage wedge of the inlet, shock waves can also be generated when $\mathrm{H}_{2}$ injects into the core airflow from the strut-injectors. These shock waves interact with each other and also interact with the main OSWs and wedge walls of the inlet, resulting in a complex inlet flow field. Three fuel layers originated from the three strutinjectors flow downstream to the end of the inlet and mix with the core airflow in the meanwhile. As indicated in Fig. 5a, good mixing 

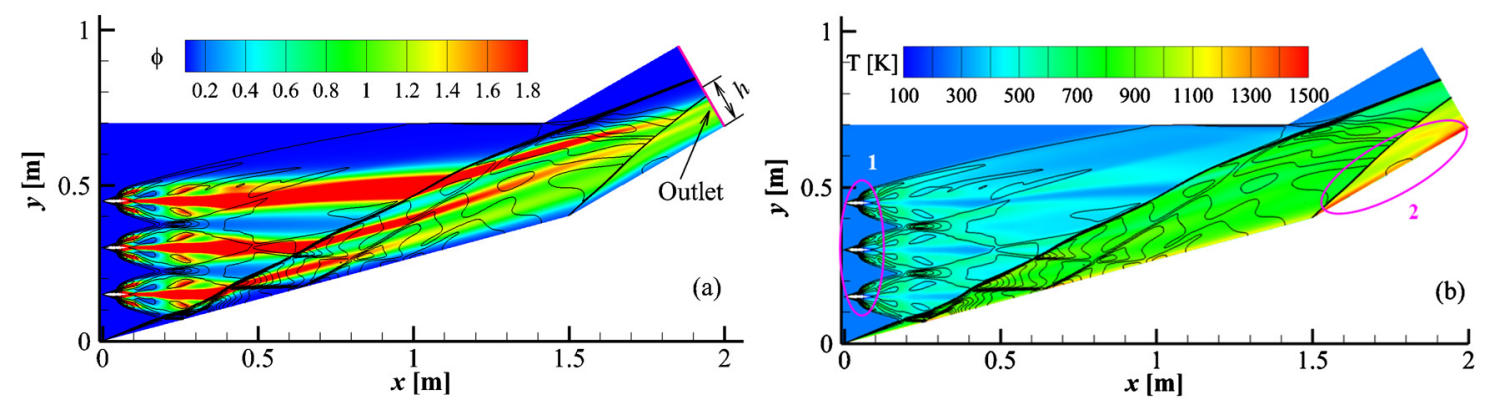

Fig. 5. Flow field of the ODE inlet at $z=0$, (a) contour of $\mathrm{H}_{2}$ equivalence ratio and (b) contour of temperature (iso-lines: pressure).

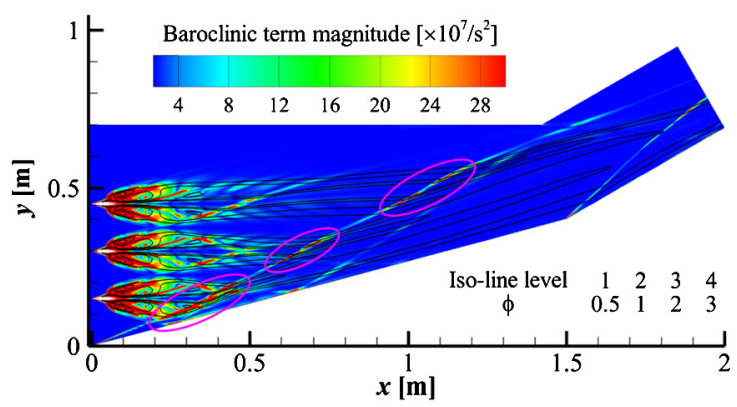

Fig. 6. Contour of baroclinic term magnitude $\left(|\nabla \rho \times \nabla p| / \rho^{2}\right)$ in the ODE inlet at $z=0$.

occurs earlier for the lower fuel layer than others. This can be attributed to the fact that the lower fuel layer meets the main OSWs earlier than others, since mixing can be enhanced by the baroclinic effect of OSWs. As indicated in Fig. 6 where the distribution of the baroclinic term magnitude $\left(|\nabla \rho \times \nabla p| / \rho^{2}\right)$ along with the $\mathrm{H}_{2}$ equivalence ratio iso-lines is shown, apart from the zones near the strut-injectors, significant baroclinic effect also occurs on the first main OSW surface when it interacts with the three fuel layers (circled by the magenta lines), which enhances the mixing of $\mathrm{H}_{2}$ and air. Moreover, the significant increase of gas density and hence the diffusion effect across these OSWs also help $\mathrm{H}_{2}$ jets to mix with air.

Additionally, other factors, corresponding to the configuration of vertical jets from the strut-injectors, should not be neglected for the achievement of a well-mixed fuel-air mixture in the inlet. Fig. 7 shows the evolution of the $\mathrm{H}_{2}$ jets in different $x$-slices from
$0.04 \mathrm{~m}$ (the injected holes) to $1.9 \mathrm{~m}$ (almost the end of the inlet). It can be seen that after the high-pressure $\mathrm{H}_{2}$ jets vertically injecting from the small holes, they fast expand into the core airflow, resulting in great penetration and consequently good mixing of $\mathrm{H}_{2}$. Fast mixing also benefits from the intensive momentum exchange between the vertical $\mathrm{H}_{2}$ jets and the crossflow. Moreover, because of the staggered configuration of $\mathrm{H}_{2}$ jets between the upper and bottom sides of the strut-injector, $\mathrm{H}_{2}$ jets on one side would diffuse into the gaps of jets on the other side, which helps to mix as well.

Further, as indicated in Fig. 7, the distribution of $\mathrm{H}_{2}$ near the strut-injectors is inhomogeneous in the spanwise direction $(z-$ direction), resulting in the strong 3D feature of the flow field in the leading front of the engine inlet. However, after mixing with air, almost homogeneous $\mathrm{H}_{2}$ distribution in the spanwise direction is obtained from the second stage of the inlet $(x=1.5 \mathrm{~m})$. Consequently, the flow field is nearly $2 \mathrm{D}$ from the tail of the inlet to the nozzle-end of the engine. Therefore, later simulations, including the pre-combustion prediction at the second stage of inlet and the combustion simulation in the ODE combustor, can be reduced to $2 \mathrm{D}$ to save computational resources, since the $3 \mathrm{D}$ reactive simulation of a large-scale model is rather expensive.

To clearly illustrate the mixing effect of the present fuel preinjection configuration in the ODE inlet, distributions of $\mathrm{H}_{2}$ equivalence ratio, pressure and temperature, perpendicular to the wall at the end of the inlet $(z=0)$, are given in Fig. 8. The detailed location of this distribution is shown in Fig. $5 a$, and $h$ is defined as the perpendicular distance from the inlet wall. It can be seen that a large well-mixed $\mathrm{H}_{2}$-air flow is obtained within the OSW in the core of the outflow, and the resulting equivalence ratio is at the designed level around 1.0. Moreover, less amount of fuel exists

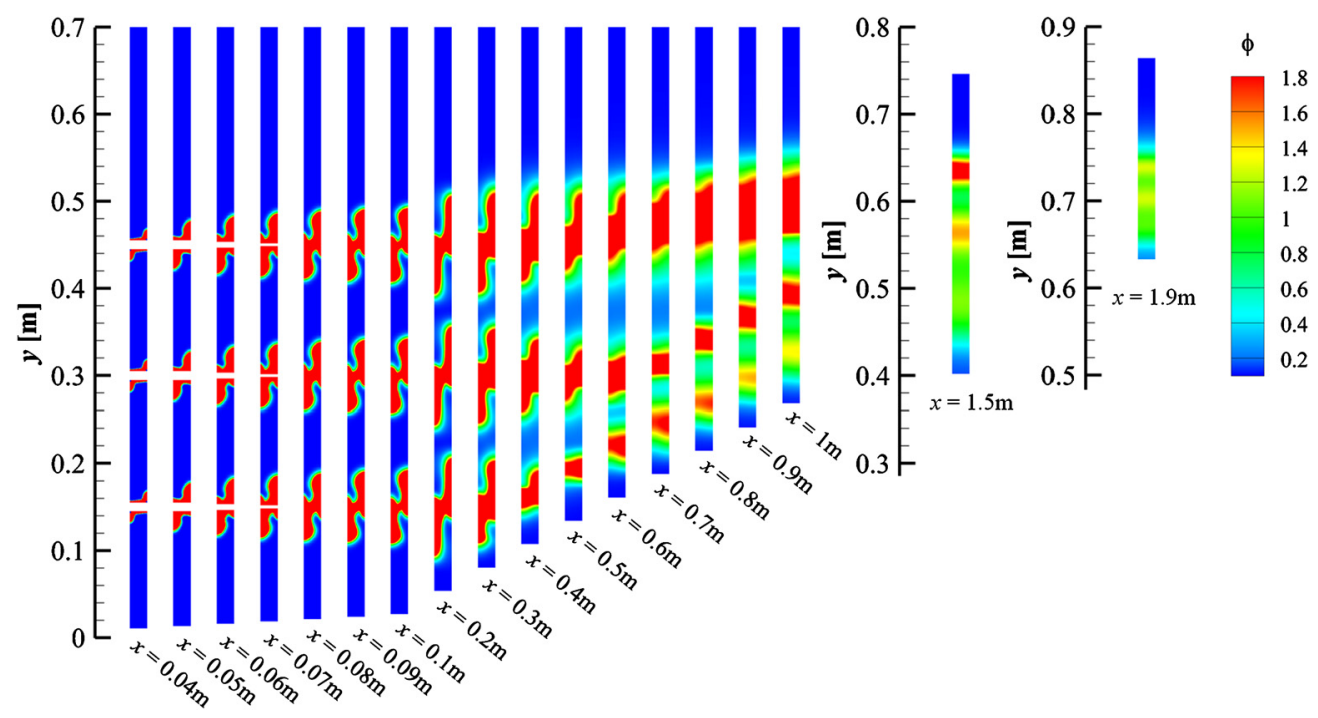

Fig. 7. Contours of $\mathrm{H}_{2}$ equivalence ratio in the inlet from $x=0.04 \mathrm{~m}$ to $1.9 \mathrm{~m}$. 


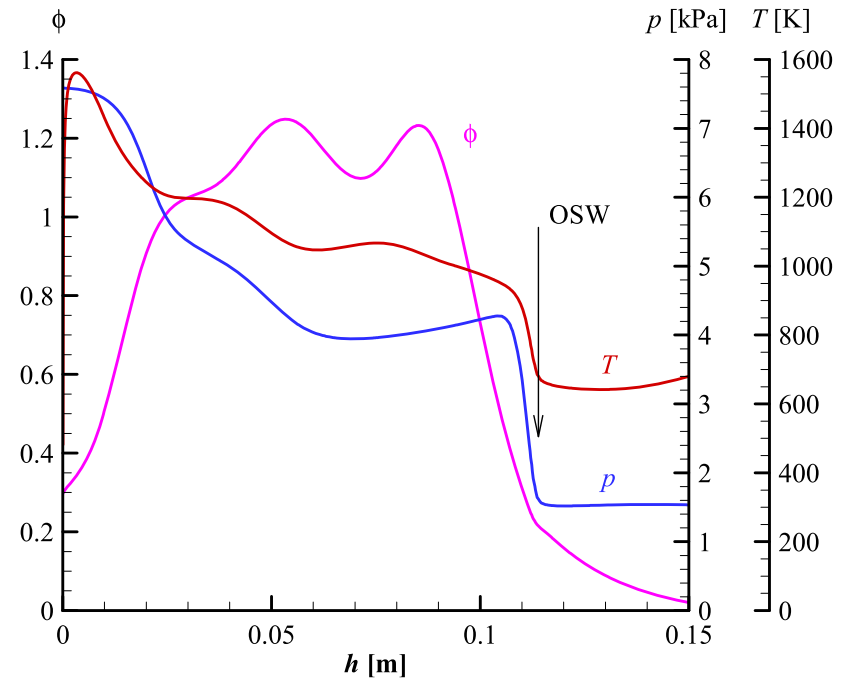

Fig. 8. Distribution of $\mathrm{H}_{2}$ equivalence ratio perpendicular to the wall at the end of the inlet $(z=0)$.

within the hot boundary layer, which is important to pre-ignition prevention in the ODE inlet. Therefore, it can be concluded that the present fuel pre-injection configuration is proved capable of providing a well-mixed supersonic fuel-air flow for the operation of the ODE.

\subsection{Pre-combustion prediction in ODE inlet}

The simulation of the 3D flow field in the ODE inlet is based on an assumption of no pre-combustion, and the success of an ODE also requires no pre-ignition existing in the inlet. Therefore, it is imperative to study the possibility of pre-ignition in the ODE inlet under the present design. As known, two factors must be satisfied for the ignition of fuel-air mixture: the mixture should reach its ignition temperature and last exceeding its ignition delay time. As for the ignition delay time, it is a function of temperature, pressure as well as the equivalence ratio of the mixture.
As indicated in Fig. 5b, there are two high-temperature zones that bear the danger of pre-ignition in the inlet. The first zone is near the strut-injectors, which is characterized by local hot spots caused by strong shock waves formed in the front of the vertical $\mathrm{H}_{2}$ jets. The second zone is at the second-stage of inlet, which is characterized by the hot boundary layer and the well mixed $\mathrm{H}_{2}$-air mixture. Some key flow parameters of these zones are summarized in Table 2, along with the local ignition delay times evaluated by the constant volume combustion assumption. The local ignition lengths are also provided and evaluated by a constant flow speed $(2500 \mathrm{~m} / \mathrm{s})$. From the estimations of local ignition lengths, danger of pre-ignition may exist near the strut-injectors.

To further analyze the possibility of pre-combustion in the ODE inlet, additional reactive simulations with the detailed Jachimowski mechanism of $\mathrm{H}_{2}$ are performed in these two most dangerous zones (3D for the zone near the strut-injectors and 2D for the zone at the second-stage of the inlet), and the results are shown in Fig. 9. Contours are given by the combustion percentage $\psi$, defined by

$$
\psi=\frac{c_{\mathrm{H}_{2} \mathrm{O}} / M_{\mathrm{H}_{2} \mathrm{O}}}{c_{\mathrm{H}_{2} \mathrm{O}} M_{\mathrm{H}_{2} \mathrm{O}}+\min \left(c_{\mathrm{H}_{2}} / M_{\mathrm{H}_{2}}, 2 c_{\mathrm{O}_{2}} / M_{\mathrm{O}_{2}}\right)},
$$

where $c$ and $M$ are the specie mass fraction and the molecular weight, respectively. $\psi=0$ means that no combustion takes place, while $\psi=1$ means that all fuel or oxidant has been reacted.

Obviously, the flow fields are characterized by rather low values of $\psi$ throughout (close to zero), indicating that no combustion occurs at these zones. As for the zone near the strut-injectors, pre-combustion prevention can be attributed to the facts that the shock waves formed in the front of the $\mathrm{H}_{2}$ jets decay rapidly downstream and that the local hot spots are so small to possess enough energy to ignite the mixture. As for the zone at the secondstage of inlet, pre-combustion prevention can be attributed to the less amount of fuel in the hot boundary layer and the consequent long ignition delay time.

\subsection{Combustion in ODE combustor}

In this section, a 2D unsteady reactive simulation of the ODE combustor and the nozzle is carried out with the detailed Jachi-

Table 2

Pre-combustion predictions estimated by constant volume combustion assumption.

\begin{tabular}{llllll}
\hline Zones & $p$ & $T$ & $\phi$ & Ignition delay time $^{\mathrm{a}}$ & Ignition length $^{\mathrm{b}}$ \\
\hline Near strut-injectors & $6.0 \mathrm{kPa}$ & $2150 \mathrm{~K}$ & 1.0 & $62 \mu \mathrm{s}$ & $15.5 \mathrm{~cm}$ \\
Second-stage of inlet & $7.6 \mathrm{kPa}$ & $1560 \mathrm{~K}$ & 0.35 & $240 \mu \mathrm{s}$ & $0.6 \mathrm{~m}$ \\
\hline
\end{tabular}

a Evaluated by a flow speed of $2500 \mathrm{~m} / \mathrm{s}$.

b Evaluated by a temperature rise of $50 \mathrm{~K}$.
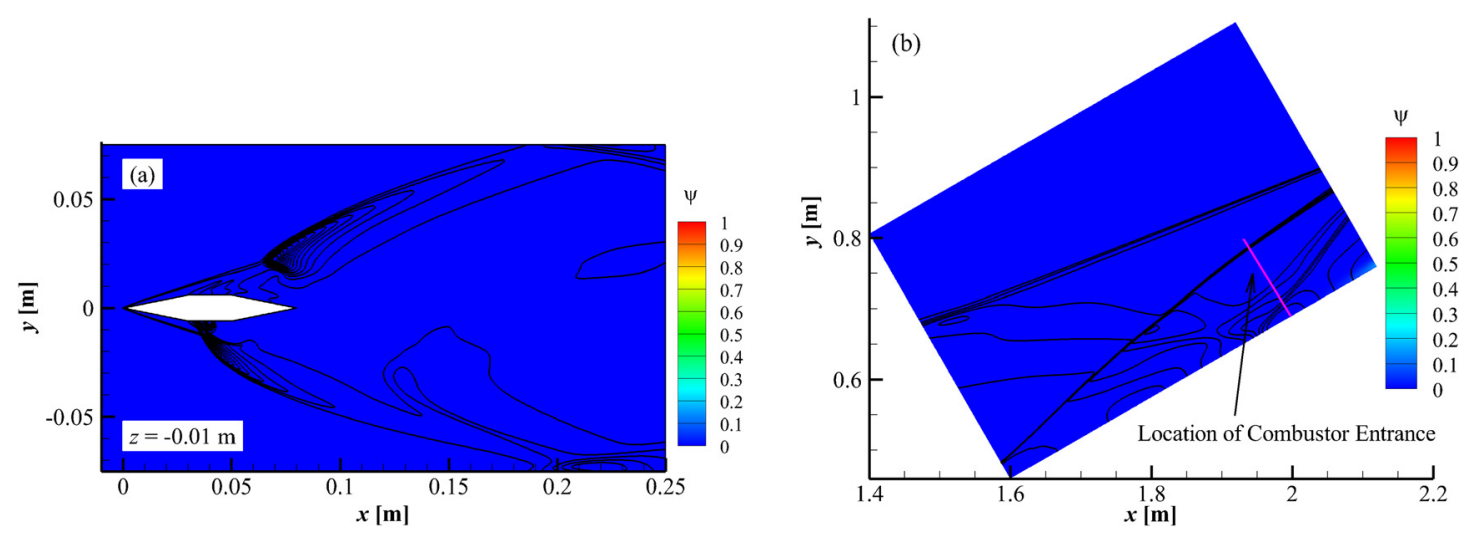

Fig. 9. Pre-combustion predictions (a) near the strut-injector and (b) at the second-stage of inlet (iso-lines: pressure). 

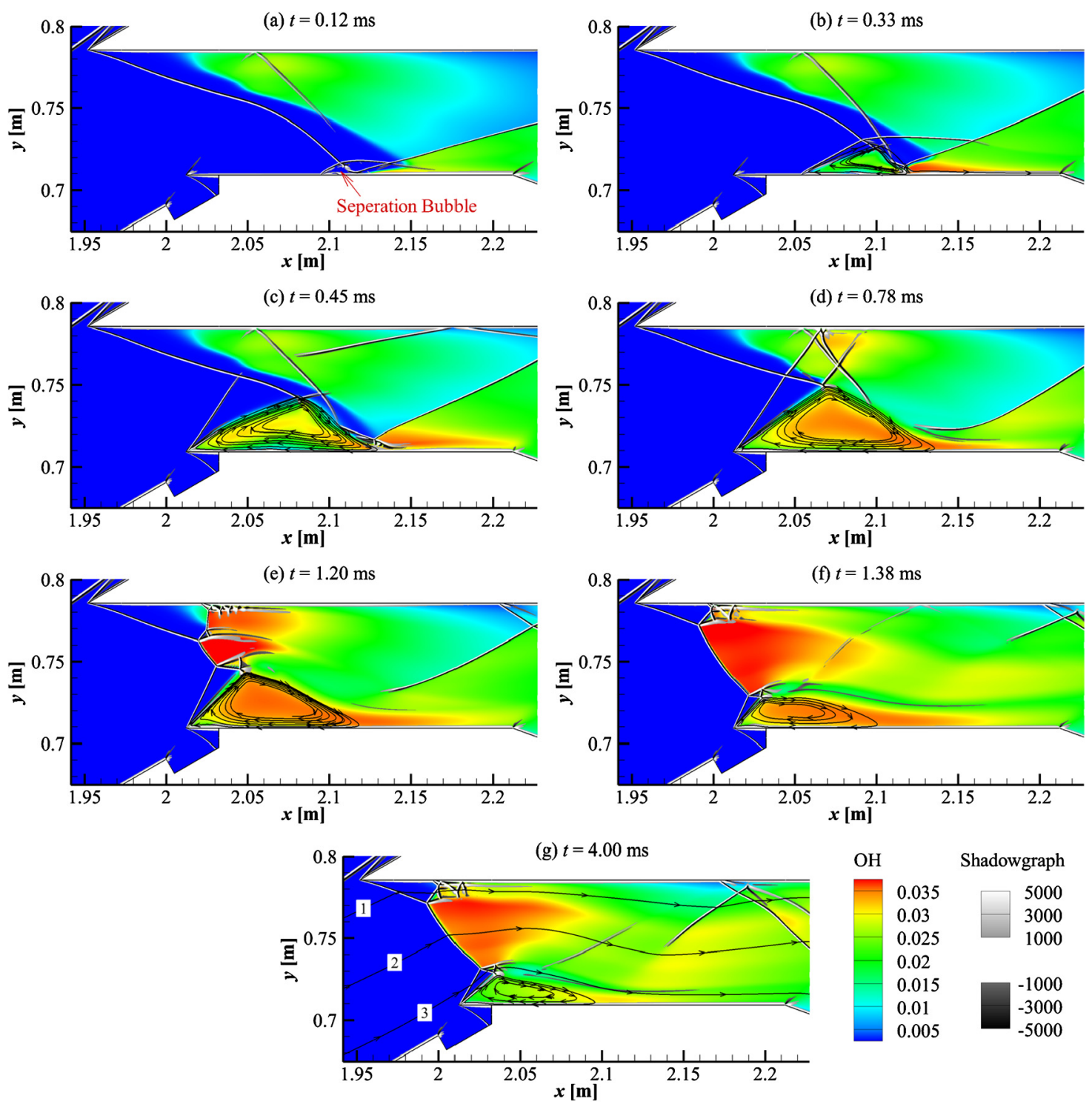

Fig. 10. Contours of $\mathrm{OH}$ mass fraction in the ODE combustor at different time instants, overlaid with middle-cut-off numerical shadowgraphs $\left(\nabla^{2} \rho\right)$.

mowski mechanism of $\mathrm{H}_{2}$. The inflow parameters are taken from the outflow of the ODE inlet in the 3D mixing simulation in Section 4.1. To control boundary layer separations in the combustor, a simple floor bleed structure is utilized at the entrance of the combustor to spill out the incoming boundary layer. The establishing process of the flow field in the combustor is shown in Fig. 10 by a serial of $\mathrm{OH}$ mass fraction contours overlaid with the corresponding numerical shadowgraphs $\left(\nabla^{2} \rho\right)$ at different time instants. The colors of the numerical shadowgraph are middle cut-off to ensure that the underlying $\mathrm{OH}$ contour could be seen where the flow field is not characterized by shock waves. At the beginning, a separation bubble is formed in the combustor when the OSW reflects at the lower wall and interacts with the boundary layer, as shown in Fig. 10a. This separation bubble moves upstream and enlarges gradually (Fig. 10b), along with the upstream movement of the separated shock wave toward the entrance of the combustor, which bears a danger of unstart. However, benefiting from the design of the floor bleed structure, the upstream movement of the separation bubble stops at the combustor entrance (Fig. 10c), as the incoming boundary layer from the engine inlet has been spilled out and the thickness of boundary layer is almost zero at the combustor entrance. Next, the separated shock wave establishes gradually and interacts with the incident shock wave to form a regular shock wave reflection pattern (Fig. 10d). Then, a Mach stem is formed and the reflection pattern transits to the Mach reflection (Fig. 10e). The size of the separation bubble becomes smaller and smaller at this time. Finally, as indicated in Figs. $10 \mathrm{f}$ and $10 \mathrm{~g}$, the flow field reaches its steady state, and the shock waves keep stable in the ODE combustor.

As indicated by the $\mathrm{OH}$ mass fraction contours in Fig. 10, combustion is first induced by the formed OSW over the cowl (Fig. 10a) and then enhanced by the reattachment shock wave formed behind the separation bubble (Figs. 10a,...,10c). As the unsteady process proceeds, locally intensive combustion occurs behind the second reflected shock wave of the cowl's OSW when the regular shock wave reflection pattern is formed (Fig. 10d). Thereafter, fast combustion is induced by the Mach stem and the reflected shock waves of the incident shock waves in the meanwhile once the Mach stem is formed (Fig. 10e). Finally, this combustion pattern remains stable along with the stable shock waves in the ODE combustor (Figs. 10f and 10g).

The final stable flow field in the ODE combustor is shown in Fig. 11 by contours of temperature and $\mathrm{H}_{2}$ mass fraction. The pressure iso-lines are also given to help analysis. Obviously, the flow 

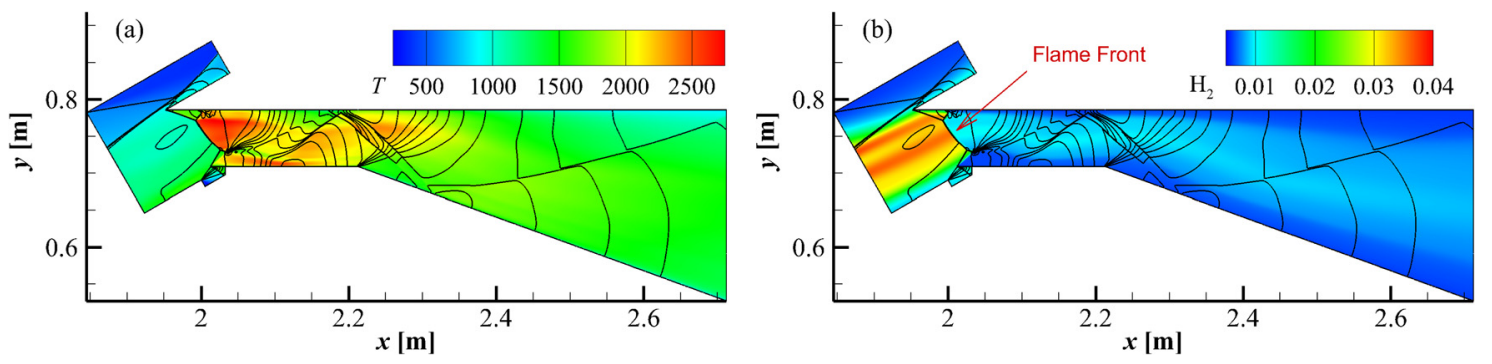

Fig. 11. Flow field of the ODE combustor and the nozzle at $t=4 \mathrm{~ms}$, (a) contour of temperature, (b) contour of $\mathrm{H}_{2}$ mass fraction. Iso-lines: pressure.
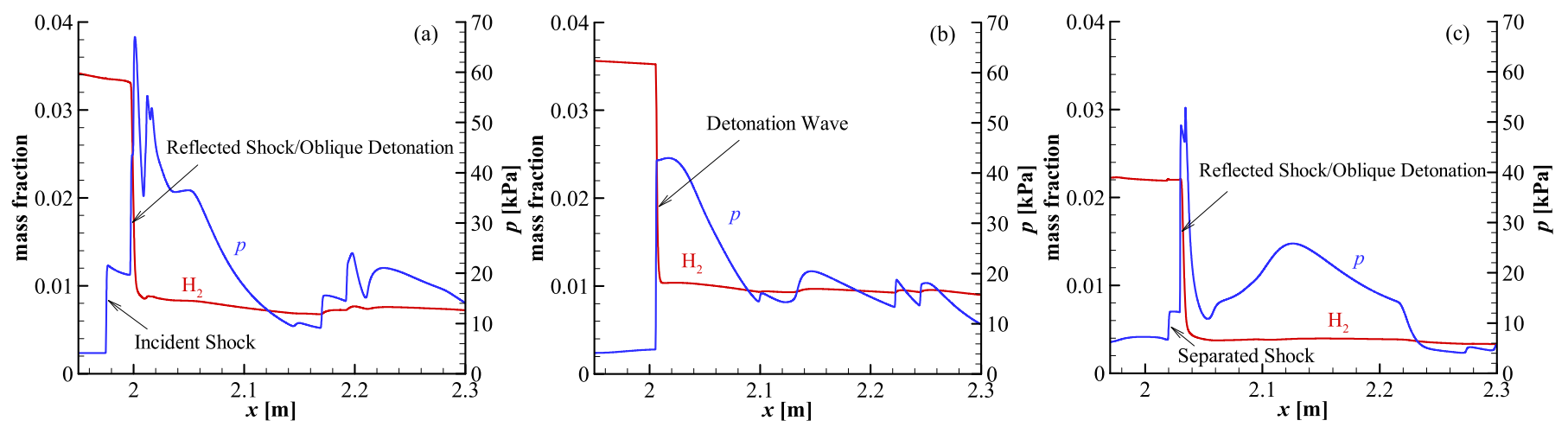

Fig. 12. Parameter distributions along the ODE combustor at $t=4 \mathrm{~ms}$, (a) streamline 1, (b) streamline 2 and (c) streamline 3 in Fig. $10 \mathrm{~g}$.

field in the combustor is divided into three parts with different shock wave structures in the height direction, which can also be observed by the numerical shadowgraph in Fig. 10g. From Fig. 11b, it can be revealed again that fast combustion is induced by these shock waves in the combustor, and the maximum combustion temperature can be achieved above $2500 \mathrm{~K}$ (Fig. 11a).

To further identify the combustion mode in the combustor, distributions of $\mathrm{H}_{2}$ mass fraction and pressure across these shock wave structures are given in Fig. 12. It can be revealed that at the location where the pressure suddenly jumps (indicating the existence of a shock wave), the mass fraction of $\mathrm{H}_{2}$ suddenly drops and then maintains at a low level, indicating the existence of a frame front where intensive combustion of $\mathrm{H}_{2}$ takes place. Combustion of the incoming $\mathrm{H}_{2}$-air mixture is achieved within these thin shock fronts, which is the typical feature of detonation waves. That is, stable detonative combustion in a confined ODE combustor is implemented in the present design.

\section{Conclusions}

In this paper, the flow fields and the performances of a Mach 9 oblique detonation engine with fuel pre-injection are numerically studied by solving the multi-species reactive RANS equations with a detailed $\mathrm{H}_{2}$ combustion mechanism. The designed ODE model is nearly $2 \mathrm{D}$ in geometry and totally about 2.8 meters in length. Three strut-injectors, locating in the leading front of the engine, are employed to supply $\mathrm{H}_{2}$ into the core airflow in the inlet directly. To control boundary layer separations in the ODE combustor, a simple floor bleed structure is utilized at the entrance of the combustor to spill out the incoming boundary layer.

The 3D mixing simulation of the ODE inlet shows that the present fuel pre-injection configuration can provide a well-mixed supersonic $\mathrm{H}_{2}$-air core flow before entering the combustor, and less amount of fuel exists in the hot boundary layer. It is shown that the good mixing can be attributed to the baroclinic effect of OSWs, the incipient expansion of fuel jets and the intensive momentum exchange of vertical jets into the crossflow. Further simulations of the two dangerous zones that bear possibility of pre- ignition in the inlet, with the use of the detailed $\mathrm{H}_{2}$ combustion mechanism, confirm that no pre-combustion occurs in the engine inlet. The 2D reactive simulation of the ODE combustor and the nozzle shows that, benefiting from the floor bleed structure, the upstream movement of the shock waves/detonation waves stops at the combustor's entrance and they remain stabilized in the combustor. Further, the pre-mixed $\mathrm{H}_{2}$-air mixture is fast burnt through the thin detonation fronts generated in the combustor. That is, stable detonative combustion is demonstrated in the ODE combustor. From the present simulation results, the previously mentioned technical difficulties of the ODEs have been numerically addressed in the meanwhile by the present design, which provides a workable solution of the pre-injection ODE for future experimental studies and engineering applications. Further attention can be paid to the optimization of thrust performance of the engine.

\section{Declaration of competing interest}

The authors declare that they have no known competing financial interests or personal relationships that could have appeared to influence the work reported in this paper.

\section{Acknowledgements}

This study was supported by the National Natural Science Foundation of China (No. 11672312 \& No. 11532014).

\section{References}

[1] D.T. Pratt, J.W. Humphrey, D.E. Glenn, Morphology of standing oblique detonation waves, J. Propuls. Power 7 (5) (1991) 837-845.

[2] Y. Zhang, Y. Fang, H.D. Ng, H. Teng, Numerical investigation on the initiation of oblique detonation waves in stoichiometric acetylene-oxygen mixtures with high argon dilution, Combust. Flame 204 (2019) 391-396.

[3] W. Han, C. Wang, C.K. Law, Three-dimensional simulation of oblique detonation waves attached to cone, Phys. Rev. Fluids 4 (2019) 053201.

[4] A.J. Higgins, Ram accelerators: outstanding issues and new directions, J. Propuls. Power 22 (6) (2006) 1170-1187.

[5] P. Wolański, Detonative propulsion, Proc. Combust. Inst. 34 (1) (2013) 125-158. 
[6] Y. Fang, Z. Zhang, Z. Hu, X. Deng, Initiation of oblique detonation waves induced by a blunt wedge in stoichiometric hydrogen-air mixtures, Aerosp. Sci. Technol. 92 (2019) 676-684.

[7] J.P. Sislian, R. Dudebout, J. Schumacher, M. Islam, T. Redford, Incomplete mixing and off-design effects on shock-induced combustion ramjet performance, J. Propuls. Power 16 (1) (2000) 41-48.

[8] J.P. Sislian, H. Schirmer, R. Dudebout, J. Schumacher, Propulsive performance of hypersonic oblique detonation wave and shock-induced combustion ramjets, J. Propuls. Power 17 (3) (2001) 599-604.

[9] T.E. Schwartzentruber, J.P. Sislian, B. Parent, Suppression of premature ignition in the premixed inlet flow of a shcramjet, J. Propuls. Power 21 (1) (2005) 87-94.

[10] B. Bomjan, S. Bhattrai, H. Tang, Characterization of induction and transition methods of oblique detonation waves over dual-angle wedge, Aerosp. Sci. Technol. 82-83 (2018) 394-401.

[11] J. Kasahara, T. Fujiwara, T. Endo, Chapman-Jouguet oblique detonation structure around hypersonic projectiles, AIAA J. 39 (8) (2001) 1553-1561.

[12] J. Kasahara, T. Arai, S. Chiba, K. Takazawa, Y. Tanahashi, A. Matsuo, Criticality for stabilized oblique detonation waves around spherical bodies in acetylene/oxygen/krypton mixtures, Proc. Combust. Inst. 29 (2) (2002) 2817-2824.

[13] A. Higgins, A. Bruckner, Experimental investigation of detonation initiation by hypervelocity blunt projectiles, in: 34th Aerospace Sciences Meeting and Exhibit, 1996.

[14] J. Verreault, A.J. Higgins, Initiation of detonation by conical projectiles, Proc. Combust. Inst. 33 (2) (2011) 2311-2318.

[15] C.I. Morris, M.R. Kamel, R.K. Hanson, Shock-induced combustion in high-speed wedge flows, Symp. (Int.) Combust. 27 (2) (1998) 2157-2164.

[16] C. Viguier, L.F. Figueira da Silva, D. Desbordes, B. Deshaies, Onset of oblique detonation waves: comparison between experimental and numerical results for hydrogen-air mixtures, Symp. (Int.) Combust. 26 (2) (1996) 3023-3031.

[17] Y. Fang, Y. Zhang, X. Deng, H. Teng, Structure of wedge-induced oblique detonation in acetylene-oxygen-argon mixtures, Phys. Fluids 31 (2019) 026108.

[18] C. Tian, H. Teng, H.D. Ng, Numerical investigation of oblique detonation structure in hydrogen-oxygen mixtures with Ar dilution, Fuel 252 (2019) 496-503.

[19] Y. Zhang, P. Yang, H. Teng, H.D. Ng, C. Wen, Transition between different initi ation structures of wedge-induced oblique detonations, AIAA J. 56 (10) (2018) 4016-4023.

[20] Y. Liu, Y.S. Liu, D. Wu, J.P. Wang, Structure of an oblique detonation wave induced by a wedge, Shock Waves 26 (2) (2016) 161-168.

[21] H.H. Teng, H.D. Ng, K. Li, C.T. Luo, Z.L. Jiang, Evolution of cellular structures on oblique detonation surfaces, Combust. Flame 162 (2) (2015) 470-477.

[22] Y. Zhang, L. Zhou, J. Gong, H.D. Ng, H. Teng, Effects of activation energy on the instability of oblique detonation surfaces with a one-step chemistry model, Phys. Fluids 30 (2018) 106110.

[23] Y. Fang, Z. Zhang, Z. Hu, Effects of boundary layer on wedge-induced oblique detonation structures in hydrogen-air mixtures, Int. J. Hydrog. Energy 44 (41) (2019) 23429-23435.

[24] Z. Ren, B. Wang, G. Xiang, L. Zheng, Numerical analysis of wedge-induced oblique detonations in two-phase kerosene-air mixtures, Proc. Combust. Inst. 37 (3) (2019) 3627-3635.

[25] Z. Ren, B. Wang, G. Xiang, L. Zheng, Effect of the multiphase composition in a premixed fuel-air stream on wedge-induced oblique detonation stabilisation, J. Fluid Mech. 846 (2018) 411-427.

[26] F.K. Lu, H. Fan, D.R. Wilson, Detonation waves induced by a confined wedge, Aerosp. Sci. Technol. 10 (8) (2006) 679-685.
[27] M.M. Roy, Moteurs thermiques, C. R. Acad. Sci. 222 (1) (1946).

[28] D.C. Alexander, J.P. Sislian, B. Parent, Hypervelocity fuel/air mixing in mixedcompression inlets of shcramjets, AIAA J. 44 (10) (2006) 2145-2155.

[29] D.C. Alexander, J.P. Sislian, Computational study of the propulsive characteristics of a shcramjet engine, J. Propuls. Power 24 (1) (2008) 34-44.

[30] J. Chan, J.P. Sislian, D. Alexander, Numerically simulated comparative performance of a scramjet and shcramjet at Mach 11, J. Propuls. Power 26 (5) (2010) 1125-1134.

[31] J.P. Sislian, B. Parent, Hypervelocity fuel/air mixing in a shcramjet inlet, J. Propuls. Power 20 (2) (2004) 263-272.

[32] Y.W. Wang, J.P. Sislian, Numerical simulation of gaseous hydrocarbon fuel injection in a hypersonic inlet, J. Propuls. Power 26 (5) (2010) 1114-1124.

[33] Y. Fang, Z. Hu, H. Teng, Numerical investigation of oblique detonations induced by a finite wedge in a stoichiometric hydrogen-air mixture, Fuel 234 (2018) 502-507.

[34] G. Xiang, X. Li, X. Sun, X. Chen, Investigations on oblique detonations induced by a finite wedge in high altitude, Aerosp. Sci. Technol. 95 (2019) 105451.

[35] M. Chapuis, E. Fedina, C. Fureby, K. Hannemann, S. Karl, J.M. Schramm, A computational study of the HyShot II combustor performance, Proc. Combust. Inst. 34 (2) (2013) 2101-2109.

[36] S. Chakravarthy, A unified-grid finite volume formulation for computational fluid dynamics, Int. J. Numer. Methods Fluids 31 (1) (1999) 309-323.

[37] E.F. Toro, Riemann Solvers and Numerical Methods for Fluid Dynamics: A Practical Introduction, Springer Science \& Business Media, 2013.

[38] F.R. Menter, Two-equation eddy-viscosity turbulence models for engineering application, AIAA J. 32 (8) (1994) 1598-1605.

[39] B.E. Launder, On the computation of convective heat transfer in complex turbulent flows, ASME J. Heat Transf. 110 (4b) (1988) 1112-1128.

[40] H. Grotjans, F. Menter, Wall functions for general application CFD codes, in: Proceedings of the 4th European Computational Fluid Dynamics Conference, ECCOMAS 98, John Wiley \& Sons, 1998, pp. 1112-1117.

[41] C.J. Jachimowski, An analytical study of the hydrogen-air reaction mechanism with application to scramjet combustion, NASA TP-2791, 1988.

[42] B.J. McBride, M.J. Zehe, S. Gordon, NASA Glenn Coefficients for Calculating Thermodynamic Properties of Individual Species, NASA TP-2002-211556, 2002.

[43] K. Wu, Investigation on the flame stabilization modes and chemical mechanism reduction in supersonic combustion, $\mathrm{PhD}$ theses, University of Chinese Academy of Sciences, 2018

[44] C. Fureby, M. Chapuis, E. Fedina, S. Karl, CFD analysis of the HyShot II scramjet combustor, Proc. Combust. Inst. 33 (2011) 2399-2405.

[45] J.Y. Choi, Computational fluid dynamics algorithms for unsteady shock-induced combustion, Part 1: validation, AIAA J. 38 (7) (2000) 1179-1187.

[46] J.Y. Choi, E.J.R. Shin, I.S. Jeung, Unsteady combustion induced by oblique shock waves at the nonattaching condition of the oblique detonation wave, Proc. Combust. Inst. 32 (2) (2009) 2387-2396.

[47] H.F. Lehr, Experiment on shock-induced combustion, Astronaut. Acta 14 (4-5) (1972) 589-597.

[48] M.C. Burrows, A.P. Kurkov, An analytical and experimental study of supersonic combustion of hydrogen in vitiated air stream, AIAA J. 11 (9) (1973) 1217-1218.

[49] Z. Jiang, H. Yu, Experiments and development of long-test-duration hypervelocity detonation-driven shock tunnel (LHDst), in: 52nd Aerospace Sciences Meeting, 2014.

[50] Z. Jiang, H. Yu, Theories and technologies for duplicating hypersonic flight conditions for ground testing, Nat. Sci. Rev. 4 (3) (2017) 290-296. 